\title{
BMJ Open Lasting impact: a qualitative study of perspectives on surgery by adult recipients of free mission-based surgical care in Benin
}

Kristin L Close, ${ }^{1}$ Floor T.E. Christie-de Jong $^{2,3}$

To cite: Close KL, Christie-de Jong FT.E. Lasting impact: a qualitative study of perspectives on surgery by adult recipients of free mission-based surgical care in Benin. BMJ Open 2019;9:e028235. doi:10.1136 bmjopen-2018-028235

- Prepublication history for this paper is available online. To view these files, please visit the journal online (http://dx.doi. org/10.1136/bmjopen-2018028235).

Received 05 February 2019 Revised 14 September 2019 Accepted 04 October 2019
Check for updates

(C) Author(s) (or their employer(s)) 2019. Re-use permitted under CC BY-NC. No commercial re-use. See rights and permissions. Published by BMJ.

${ }^{1}$ Department of Public Health, University of Liverpool, Liverpool, UK

${ }^{2}$ Department of Public Health, Faculty of Health Sciences and Wellbeing, University of Sunderland, Sunderland, UK ${ }^{3}$ Department of Public Health, University of Liverpool Faculty of Health and Life Sciences, Liverpool, UK

Correspondence to

Dr Kristin L Close;

closekristin18@gmail.com

\section{ABSTRACT}

Objectives This study aimed to explore how adult patients who received free mission-based elective surgery experienced surgery and its outcomes, in order to provide recommendations for improved service delivery, measurement of impact and future quality initiatives for the humanitarian organisation Mercy Ships and other mission-based surgical platforms.

Setting Data were collected in June 2017 in Cotonou, Benin, where the participants had previously received free mission-based elective surgery aboard the Africa Mercy, a non-governmental hospital ship.

Participants Sixteen patients (seven male, nine female, age range 22-71, mean age 43.25) who had previously received surgical care aboard the Africa Mercy hospital ship between September 2016 and May 2017 participated in the study. Methods Using a qualitative design, 16 individual semistructured interviews were conducted with the assistance of two interpreters. Participants were recruited using purposive sampling from the Mercy Ships patient database. Interview data were coded and organised into themes and subthemes using thematic content analysis in an interpretivist approach.

Findings Analysis of interview data revealed three main themes: barriers to surgery, experiences with Mercy Ships and changes in perspectives of surgery after their experiences. Key findings included barriers to local surgical provision such as cost, a noteworthy amount of fear and distrust of local surgical teams, exceptional positive experiences with the care at Mercy Ships, and impactful surgery, resulting in high levels of trust in foreign surgical teams.

Conclusions While foreign surgical teams are meeting an immediate need for surgical care, the potential enduring legacy is one of trusting only foreigners for surgery. Patients are a critical component to a well-functioning surgical system, and mission-based surgical providers must formulate strategies to mitigate this legacy while strengthening the local surgical system.

\section{BACKGROUND}

In 2010, conditions requiring surgery caused nearly $33 \%$ of global deaths, but only $6 \%$ of the 313 million surgeries performed annually occurred in the poorest countries. ${ }^{1}$ Local surgical provision in low-income and

\section{Strengths and limitations of this study}

- This study presents a qualitative exploration of patient experiences and perspectives on the impact of surgical missions

- The sample included under-represented key stakeholders of surgical missions: the patients.

- Data were collected in the participants' preferred language and the use of professional translators may have led to some loss of the richness in the qualitative data, although the three-way conversation used in the interviews added to the credibility of the data and rigour of the study.

- Although familiar with the culture, the positionality of the primary researcher as a foreigner and outsider, and previously involved with Mercy Ships, possibly introduced response bias among participants.

- Participants were self-selecting and therefore the sample may not reflect all patients' experiences and perspectives. For example, individuals with poor experiences of the mission or individuals who may have been too fearful of surgery may not have volunteered to take part in the study.

middle-income countries (LMICs) has been shown to be critically insufficient to meet the needs of the population. ${ }^{2}$ Even if local surgical provision is available, several barriers to meeting the surgical need exist, including a lack of delivery system, equal patient access to that system, safety within the system and protection from catastrophic financial loss. ${ }^{1}$ There is a significant shortage of trained surgeons, obstetricians and anaesthesia providers in $\mathrm{LMICs}^{3}$ and a lack of essential equipment for those providers to safely perform surgery. ${ }^{4-6}$ The 2015 Lancet Commission on Global Surgery report drew international awareness to global surgical need ${ }^{1}$ and led to widespread recommendations for extensive growth and funding for surgical training, infrastructure, technological innovations and advocacy to strengthen surgical systems in LMICs. ${ }^{7}$ 
One widely implemented temporary solution to address the unmet surgical need in LMICs is surgical provision by charitable platforms or short-term medical missions (STMMs), defined as 'travel by a group of physicians to a foreign country for the purpose of making a special study or of undertaking a special study of a short-term duration ${ }^{8}$. Shrime et at offered a classification scheme of STMMs and suggested two main categories of STMMs based on the method of surgical delivery: (1) Temporary surgical platforms: surgical delivery, which can be divided into (a) short-term surgical trips, where institutions in high-income countries send healthcare professionals to LMICs' clinics or hospitals for short periods of a few weeks, or (b) selfcontained surgical platforms, which usually spend more time within an LMIC, from months to years, contain their own infrastructure, and usually do not leave behind any physical structure. (2) Specialty surgical hospitals, a model for improving surgical delivery by developing local providers and infrastructure, specialising in one or a few surgical conditions, with a combination of foreign and local staff, usually funded with foreign aid. ${ }^{9}$

STMMs' goals vary by organisation, length, specialty and purpose, from surgical provision alone to capacity building and donations; however, integration of the local healthcare system and sustainability of care should be a key aim of all STMMs. ${ }^{10}$ STMMs' efficiency, ethics and clinical benefit have been questioned ${ }^{11}$ and STMMs have been criticised for not addressing the root cause of the problem, namely, poverty, leading to under-resourced and overstretched healthcare infrastructures. ${ }^{10-12}$ Critics of STMMs have also pointed at a common lack of cultural understanding and unintended consequences of STMMs, such as the lack of follow-up and questionable role of minimally trained students or volunteers. Carlson et al discussed predominant approaches of international non-governmental organisations and Ministries of Health towards mitigating the surgical workforce crisis: expanding training for local clinicians and temporary expansion of workforce through STMMs. ${ }^{13}$ The authors discussed ethical implications of choosing one approach over the other and concluded that both are required, and emphasised that STMMs can distort the local market with regard to providers and services.

Although evaluation of STMMs still seems in its infancy, self-contained temporary platforms and specialty surgical hospital have been found to provide the most effective, cost-efficient care. ${ }^{912}$ The impact of STMMs on the population, however, beyond provider experiences, ${ }^{14}$ clinical results or patient reported outcomes, ${ }^{15}{ }^{16}$ is rarely explored, especially from a patient perspective. Evaluation of healthcare and surgical provision in high-income countries is increasingly based on patient perspectives, as patient-centred care has been shown to improve outcomes. ${ }^{17} 18$ This type of healthcare evaluation, however, is not widely practised or reported for STMMs in LMICs. While White et al quantitatively measured overall satisfaction of mission-based surgery, ${ }^{19}$ there has been limited qualitative research regarding experiences and perspectives of surgery among recipients of mission-based surgical provision. ${ }^{12} 1620$

Understanding barriers to accessing and using local surgery, experiences of surgery and subsequent patient perspectives and attitudes about surgery from recipients of STMMs can help direct future quality initiatives and ensure appropriate levels of patient satisfaction. Patient experiences and perspectives are also important to organisations seeking to encourage local sustainability, by ensuring patient-centred outcomes align with the organisations' anticipated or expected impact and are not fuelling a continued legacy of dependence on foreign investment and provision.

Mercy Ships falls in the category of a self-contained surgical platform and operates the largest civilian hospital ship in the world, the Africa Mercy, that travels to a different country in Africa every year providing free surgery and medical training by a crew of voluntary, highly trained specialised medical and support staff. The Africa Mercy ship is a state-of-the-art surgical hospital with five operating rooms, 200+volunteer medical staff and all the ancillary services required for patient care presurgery and postsurgery, including radiology, physiotherapy, access to pharmaceuticals and attentive nurses specialised in critical care, paediatrics and other specialties aboard the ship. Patients and caregivers are also given three wellbalanced meals per day, clean linens and activities for entertaining themselves or their children, which is rare or non-existent in the local context. Mercy Ships provided free surgery and postsurgical care to patients in need alongside mentoring programme, skills training and courses for local surgeons, nurses, anaesthesia providers and other surgical professionals in Benin, from August 2016 to June $2017 .{ }^{21}$ In this paper, we present results of a qualitative study that aimed to explore perspectives of surgery held by recipients of mission-based surgical provision on the Africa Mercy in this period to gain an in-depth understanding of decision-making regarding surgical provider, barriers and facilitators to local surgical provision, patients' perspectives and experiences with missions, and how those experiences affect future care-seeking behaviour, to inform future programmatic development.

\section{METHODS}

The study employed a qualitative design to explore perspectives and experiences of surgery among adult recipients of free mission-based elective surgery in Benin. The Africa Mercy performed 1957 surgeries on 1793 patients during the 2016-2017 field service. ${ }^{21}$ This study began 4 weeks after the ship departed Benin, following its 9-month service in the country, meaning at the time of invitation, potential participants had been discharged anywhere from 4 weeks to 8 months.

Telephone contact information for potential participants was collected from Mercy Ships patient database and purposive sampling was applied in relation to gender, age, location and type of surgery. The potential 
participants' surgery had to be available locally in Benin to explore decision-making processes in-depth, so patients who underwent burn contracture release surgeries, for example, were excluded from the study as this surgery is not available in Benin. Patients who underwent gynaecological and groin surgery were also excluded from the study to avoid potential culturally inappropriate discussions within a mixed-gender research team. Potential participants had to live within Cotonou or the surrounding area, ensuring they had physical access to local surgery. From the remaining patient list, the research team began contacting potential participants. To avoid any perception of pressure, potential participants were contacted by interpreters independent of the foreign researcher. They were informed that the study aimed to explore how patients felt about surgery in general and their experience on the ship, and asked if they would like to participate. In total, 30 participants were contacted and 16 participants ( 7 male, 9 female, age range 22-71, mean age 43.25) living in Cotonou, Benin, who received surgery on the ship, agreed to take part in the study. No potential participants declined or were excluded; the remaining 14 individuals were either travelling or unavailable. Participants underwent a variety of surgeries, including cataract (4); goitre (2); facial (2), neck (3), back (3) or other (1) tumours; and thumb duplication (1).

Semistructured interviews were conducted in June 2017. Participants chose their interview location; most interviews were held in participants' homes, with a few in a nearby café chosen by the participant. Interviews were conducted by the principal researcher accompanied by trained interpreters, in the participants' preferred local language (Fon, Goun and Yoruba). Participants were asked about any previous experiences of surgical care and their experiences on the ship. All interviews were, with permission from participants, recorded, translated and transcribed verbatim to English. Translations were validated by interpreters for accuracy.

Towards the end of data collection, new data repeated what participants expressed in previous interviews and no new themes were emerging, therefore no further interviews were conducted. Thematic content analysis was applied to analyse data, to get an overall feeling for the data by reading and re-reading the transcripts. Codes, or key words, were noted in the margins, followed by a focus on finding meaningful elements in each transcript. These meaningful elements from all transcripts were then grouped together to identify patterns or themes in the data. The first author started this process which was reviewed by and discussed with the coauthor. After careful deliberation, three main themes, each with subthemes, were identified.

\section{Ethical considerations}

In accordance with ethical procedures, participants received a Participant Information Sheet outlining the purpose of the study, which was explained verbally. Participants were informed that the primary researcher previously worked aboard the ship as a volunteer, was not an employee and no longer affiliated with Mercy Ships, and at the time was a Master of Public Health student at the University of Liverpool. Participants were encouraged to express themselves freely and informed that their identity would be kept entirely confidential. The team informed all participants that Mercy Ships would not be returning to Benin in the foreseeable future, and their participation would in no way affect any future interactions with the ship. All participants were encouraged to share any concerns and ask questions, while given the opportunity to decline or stop participation at any time. Written informed consent was obtained from each participant prior to the start of each interview with a thumbprint acceptable in lieu of a signature in cases of illiteracy. No identifying information was included in recorded interviews. The interpreters were trained in medical confidentiality and this was reinforced throughout the study.

\section{Patient and public involvement statement}

Although the study aimed to provide a platform for patients' perspectives of free mission-based surgical care in Benin, patients and the public were not involved in the design of the study. There are no plans to disseminate the study results to patients due to language and logistical barriers.

\section{FINDINGS}

Three distinct themes emerged: (1) barriers to local surgery, (2) personal experiences and impact surgery had on participants' lives and (3) changed perspectives of surgery and advice they would give to others regarding surgery.

\section{Theme 1: barriers to local surgery}

Although all participants had access to local surgery to address their condition, few had sought medical or surgical consultations, and even fewer received any treatment. Exploration of these experiences revealed a strong theme of significant barriers to obtaining the surgery needed, with subthemes of cost, lack of awareness and consideration of local surgical provision, fear of local surgical provision and cultural beliefs.

\section{Cost}

Cost was raised as a barrier to local surgery by six patients, but it was never the primary or first reason given. Four participants specifically noted, without prompts, that they were unable to afford either a local consult or surgery, and therefore did not proceed with treatment. Half of these participants knew the approximate cost of treatment, while the other half had just heard it would be unreasonably expensive.

I remember one of the doctors said I should give one million CFA [local currency, approximately \$1500] before having it removed, and somewhere else they said I should buy a whole box of blood before I had the goitre removed, and then paid some money... I've been told I need to buy lots of medicine... so I've 
been discouraged by all the things I've heard so far. (Participant 11, F, 46, goitre)

Lack of awareness and consideration of local surgical provision Some participants expressed they had limited knowledge of local surgical provision or demonstrated a lack of information provided by healthcare providers. Five participants had never considered getting a medical or surgical consultation because they felt the problem (the tumour or cataract) was not having a significant negative impact on their lives. Other participants reported consulting with a local provider but were told local treatment was not available.

My parents told me when I was still a young boy they took me to [local hospital] but they were told that [the tumour] couldn't be helped. (Participant 8, M, 40 , back tumour)

\section{Fear or distrust of local surgical provision}

Some participants consulted with local surgeons, expecting a surgical intervention, but they were given non-surgical treatments that did not help. This seemed to lead to a distrust of local providers who were unable to improve their condition. One participant shared: "they told us they saw nothing wrong with the eye, but they prescribed some eye drops that we bought and we used, with no special result" (Participant 13, F, 22, cataract). Six participants had experienced a previous surgical procedure: three were emergency procedures performed by local surgeons, unrelated to participants' Mercy Ships-treated condition; and three were elective procedures, one by a local surgeon and two by foreign surgeons using local nurses in a local hospital. Two of the elective procedures were for conditions later treated by Mercy Ships. All participants shared difficult stories of being treated poorly or experiencing unfavourable outcomes as a result of the surgery. Participants shared common views of not feeling well-received or cared for; they felt local medical teams showed a lack of respect towards patients and rarely informed them of what to expect throughout the process. Patients were required to provide all their own meals, often left alone in pain, and shared a general belief that local teams were unconcerned with whether patients lived or died.

It is when I went to [local hospital] that I had that very bad experience that I decided not to trust them anymore. I was really afraid for they gave me anaesthesia, they left me there for some while... and it was not successful, you know all these things made me to be really afraid of going back to [them]. (Participant 5, M, 48, facial tumour)

Nine participants, while not having previous surgical experiences themselves, knew of, or attended to, close relatives or friends who had undergone a surgical or medical experience that left them fearful and untrusting of local providers. Three participants shared they would not go for consultation with local surgeons because they believed that the surgeon would only make the condition worse. Three participants referred to people with the same condition as theirs who died in surgery. Another participant shared a story of an ill child not being well received by a local team; the child sadly died, and the participant vowed never to trust local providers again.

They didn't do well with us, they didn't receive us well, they wasted our time, they didn't take good care of the child... they waited until the situation got very worse before they sent us to [the referral hospital]... the child didn't survive. (Participant 4, F, 49, neck tumour)

While much distrust to local surgical provision was the result of experiences, several references were also made to seemingly generally accepted rumours or beliefs about poor hospital care, which were not tied to a specific person or story. These stories often began with 'everyone knows', 'people say', or 'many people believe'. A higher level of trust towards white, foreign or 'yovo', surgeons was suggested, with several participants sharing stories of having consulted only with foreign surgical teams visiting the country. This trust in foreign surgeons was reflected in the higher level of trust these participants were willing to give to the Mercy Ships team; while most noted they were somewhat afraid of the surgery itself, that fear did not keep them from accessing the services in the way it kept them from approaching a local surgeon.

People used to say when yovos perform surgery on you, you'll be okay. (Participant 1, M, 41, back tumour)

\section{Cultural and religious beliefs}

Cultural and religious beliefs also emerged from the data as reasons why participants chose not to trust local providers for their surgical care. Several participants expressed feeling guided by God in their decision to go for surgery and some believed the ship coming to Benin was a gift from God.

I used to hear people saying, he's a Christian but still he's carrying that big tumour around. And I used to tell them the Lord is able. And I think the last one that I said, after saying that it didn't take three months before mercy ships came. (Participant 6, M, 71, neck tumour)

Many shared fatalistic beliefs that having surgery often meant dying or leading to worse outcomes than participants experienced before surgery.

We hear of people going in for surgery and coming out of it dead. So you know, generally speaking, people are afraid of going to surgery. (Participant 8, M, 40 , back tumour)

Most of the time, people who have gone through eye surgery... they are blind today. (Participant 12, F, 40, cataract) 
One participant shared he had seen a local surgeon for a consultation and had an appointment for surgery but expressed: "in my spirit I felt I couldn't go to the local surgeon or I would die" (M, 71, neck tumour). Others shared similar experiences, one feeling 'in her heart' that she should not have the scheduled surgery, and another stated 'his spirit' said not to go for surgery. Two participants' families encouraged them to seek care from traditional healers, suggesting they were more trustworthy than medical doctors. Both did so under pressure from family, but shared that treatment had no impact on their condition. Among all participants, there were no evident strong feelings for or against local healers and remedies, but this was not explored extensively.

\section{Theme 2: experiences with Mercy ships}

All participants were eager to discuss experiences with Mercy Ships; none of the participants reported any negative experiences or suggestions for improvement, and all shared they were pleased with the result of their surgery. Many patients shared they were initially still afraid to have surgery, but much less so as they knew or had heard of people who had good experiences with Mercy Ships, or they had seen or heard advertisements on television or radio that encouraged them to try. When discussing their experiences, two predominant subthemes emerged: experience of caring treatment and the impact surgery had on their lives.

\section{Caring treatment}

Most participants explained how, in their view, Mercy Ships was different from local hospitals. Participants expressed that Mercy Ships nurses 'felt like family'; they welcomed patients with a smile and cared for them with respect and dignity. Several participants expressed gratitude that Mercy Ships staff explained what was going to happen, which, according to participants, did not often occur in local hospitals.

And there is always someone to prepare for you and answer your questions...in our hospitals here, it's very different. (Participant 11, F, 46, goiter)

Anything [Mercy Ships] want to do to you they will explain it to you, they will tell you the steps you are to take now, the stage you have got to, they will tell you everything and prepare you mentally and psychologically towards what is going to happen. (Participant $16, \mathrm{M}, 45$, cataract)

Participants expressed how grateful they were to have food provided, as locally, patients or the patient's family are responsible for providing meals. Many participants shared they never wanted to leave the ship. They also noted nurses wanted to alleviate pain if possible, which they believed was not common in local hospitals. Many participants perceived a difference in the amount of training Mercy Ships or foreign surgical teams received and the standards to which they held their practice.

I think in our local hospitals, there is a lack of professional conscience... which is not the same with Mercy
Ships people. Everything has to be at a certain standard, and people have to be chosen, based on their skills, and the way they are organized, the way they perform. (Participant 14, M, 51, back tumour)

\section{Impact}

For many participants, successful surgery meant being able to work and support their families again. Participants reported less pain, itching, fatigue and other debilitating problems that had plagued them. For others, it meant going into public unashamed and unhindered. In Benin, a tumour can be viewed by some as a curse or a sign the person is performing witchcraft. Removal of the tumour removed the shameful shadow that some of these participants lived in.

I think this has... had a good impact on my family relationships as well... when we go for family meetings or funerals or anything somewhere, people will be asking you what is wrong with you... now no one will ask me that anymore, so it makes my relationship with people very simple, and nice, and stress-less. (Participant 2, M, 27, facial tumour)

You know we live in Benin and we are in Africa, when people are seeing such a thing on your head they will say you are hiding your witchcraft calabash there. (Participant 7, F, 56, neck tumour)

\section{Theme 3: changed perspectives of surgery}

All participants shared that their experiences with Mercy Ships changed how they felt about surgery in general. While fear was the common emotion going into surgery, after their Mercy Ships experiences, participants seemed to agree that surgery was not something to be feared. However, many of these sentiments towards surgery included a qualifier; they were no longer afraid if it was Mercy Ships performing the surgery. Participants often indicated they would still fear surgery with a local surgical team.

After this experience, I could accept, I could say to myself, that people can decide to go to surgery without fear. Because I've had the experience, and I've come out well, so I told myself so when people say I'm going for operation, that doesn't mean they are going to die. So I think it's not a bad thing to go for surgery. (Participant 3, M, 24, thumb duplication)

When discussing advice they might offer family members or friends who were facing a similar situation, participants overwhelmingly responded they would advise others to wait for Mercy Ships to return. Some, recognising that Mercy Ships missions to a particular country are not regularly scheduled, would suggest others only see foreign surgical missions that come to Benin on a regular basis. Finally, a few participants did suggest they might consider recommending local surgeons who had been trained by Mercy Ships, but only as a last resort.

If I hear about one of our Beninese doctors who has been trained by the ship, who is doing well in [local 
hospital], I might advise my brother or my cousin to go there, but still, if the next coming of the ship is not far, I will rather advise my brother to wait until the yovo team comes. (Participant 1, M, 41, back tumour)

\section{DISCUSSION}

Themes presented in the results are intertwined and together lead to several implications for the field of global surgery. The surgery these Mercy Ships patients needed was locally available to them, but they did not pursue it because of cost, lack of awareness and consideration of local surgical provision, fear or distrust, and cultural beliefs. Participants went to Mercy Ships for surgery, most still feeling afraid but less so, and had an experience quite contrary to what they had expected in the local context. These patients thus expressed changed perspectives about surgery, and they would advise others to wait for Mercy Ships or another foreign STMM. Positive views on surgery came with a significant caveat, that the surgical team needed to be a foreign team.

Access and financial barriers to surgery have been reported in the existing literature. Lin et al surveyed 1237 Mercy Ships patients in Congo, and cost was cited as a barrier to surgery by $73 \%$ of patients, followed by $8 \%$ indicating no surgeon was available to perform the needed surgery. ${ }^{22}$ Cost was cited as a barrier in the current study and participants also shared how much they appreciated additional elements to their healthcare at Mercy Ships, such as food and accommodation for relatives. Inclusion of these ancillary cost into their care seemed to impact patient experiences positively. However, although cost was reported as a barrier, it was cited infrequently relative to experiences participants shared that led them to fear local hospitals. Only 12 patients $(0.009 \%)$ in Lin et als sample reported feeling afraid or untrusting of local surgeons, ${ }^{22}$ which seems to contradict findings in the current study of fear and distrust as a barrier to surgery. However, this contradiction may be explained by Kessy and Lewallen, ${ }^{23}$ who studied patients with cataract who declined surgery in Tanzania. The authors suggest that prohibitive costs of surgery often serves patients as a 'convenient and acceptable explanation that will not be challenged by health workers'. ${ }^{23}$ This could account for the indication of cost as a primary barrier in the Lin $e t a t^{22}$ survey, but if responses of patients and experiences were explored in more depth as in the current study, one may find cost was one explanation but may not wholly reflect barriers encountered by patients. Grimes et al highlighted the difficulty in measuring the relative importance of various barriers in their systematic review of barriers to surgery in LMICs, noting barriers are intertwined and ranking them is complicated; they suggest that, from a patient perspective, a barrier is a barrier, and one is not necessarily worse or more significant than another. ${ }^{24}$ Due to this difficulty and the multifactorial influences of various barriers to surgery, providers and systems strengthening initiatives must also consider multifactorial solutions to eliminating these barriers.
Exploration of barriers to surgical care, specifically in LMICs, has shown a multitude of components that constitute access. The Lancet Commission on Global Surgery highlighted that 5 billion people who lack access to surgical care face multidimensional barriers including a lack of delivery system (structure), access to that system, safety within the system and financial protection. ${ }^{1}$ While this description of access is certainly valid, even if all those missing components were in place, patients might still not be willing to access care; patient fears and cultural beliefs also need to be taken into consideration.

In this study, all participants lived within $30 \mathrm{~min}$ of Cotonou, where appropriate surgery was available to them, effectively eliminating some access barriers highlighted as critical deficiencies in surgical systems by Meara et al. ${ }^{1}$ While measurement of the actual safety of the local surgical structure was not a part of this study, results suggest that perceived safety within the local surgical system is a critical component to patient uptake of services. Many participants indicated they felt local teams lacked skills and feared having surgery would result in death; this perception of insufficient safety in surgery is based on stories and experiences, not on empirical measurements of actual surgical system safety in Cotonou hospitals. However, fear of surgery is a known barrier to care, with women over three times more likely than men to experience such fear. ${ }^{25}$ Mercy Ships' screening teams have developed rural screening strategies, shown to reduce some of these fears for patients who received surgery in Madagascar. ${ }^{26}$

While STMMs can affect change in a few hundred or thousand patients, the legacy among the greater population seems a potential dependence on and development of trust in foreign surgical providers, possibly connected to a distrust of local providers. The potential enduring legacy of STMMs is one of trusting only foreigners for surgery, possibly leaving behind a legacy of distrust and fear. Millions of dollars are funnelled into strengthening surgical systems in LMICs; roadmaps for high-income countries have been suggested ${ }^{7}$ and implemented, including massive investments in training, infrastructure and equipment. ${ }^{27}$ However, patient perspectives have not been considered; the implications of this research suggest even if systems were in place and local hospitals were performing safe, accessible and affordable surgery, patients may still be unwilling to consider local surgery. Mission-based surgical providers need to consider ways to mitigate this legacy of distrust of local providers. Sustainability of care and capacity building must be key goals of each STMM.

The burden of unmet surgical need is significant, particularly in LMICs, where an estimated $94 \%$ of the population cannot access safe, timely and affordable surgery. ${ }^{1}$ As many non-profit organisations, foundations, charities and governments work independently and together to address this public health crisis, patient perspectives must be taken into consideration and barriers to surgery addressed. Findings of this study highlight perspectives of patients influenced by mission-based surgical provision as unfavourable towards local providers and services. Organisations 
working to strengthen these local providers and services must take this into consideration as they develop training programme and systems-strengthening initiatives, to influence the perceptions of the patients with regard to surgery and surgical systems. For any surgical systems strengthening programme to be effective in lowering the burden of surgical disease, patients must be willing and able to receive provided services. Trust is a key component to patient careseeking behaviour ${ }^{28}$ and favourable outcomes improving the populations' trust towards local surgery teams should be an integral part in any capacity-building or development programme in LMICs.

This study found overwhelmingly positive views of their surgery and care with Mercy Ships, perhaps not surprising, since in the Africa Mercy hospital, patients experience a 1:3 nurse to patient ratio; in local hospitals, this can be as much as 1:30. In addition, patient care in local hospitals can be extremely expensive and patients are often required to purchase all medical supplies in addition to paying for clinical care and bed space. ${ }^{29}$ Patients in the Africa Mercy hospital pay for nothing and are given enough disposable supplies to continue any lingering wound care after discharge. Patient attitudes of appreciation are likely influenced not only by the care they received but also the knowledge this experience will not cost them their home or livelihood. Participants used expressions of gratitude and voiced no criticism of their care. Although the research team aimed to reassure participants that there were no right and wrong views and that all views would be acceptable, it cannot be known whether participants may have felt reluctant to express negative views. A limitation to the study may be the positionality of the first author as a foreigner and previous volunteer with Mercy Ships, which may have induced bias in participants seeking to give only good feedback to the organisation out of a sense of gratitude or obligation. To mitigate against any perceived power imbalance, the researcher was introduced as a research student, not a Mercy Ships volunteer, and interviews took place after the Mercy Ships mission to Benin had completed. The researcher travelled with two Beninese counterparts for interpretation, safety and cultural assistance; every interview was discussed while reflecting on any potential expressions of bias, power imbalance or other influence on the trustworthiness of the data collected. Reflexivity continued throughout the analysis. However, these exceptionally positive attitudes to STMMs and a sense of gratitude have also been reported in the literature for aid recipients. ${ }^{160}$ Roche et al described a potential power imbalance for aid recipients of STMMs, possibly reflecting recipients' inability to navigate, access or afford local healthcare, leading to a sense of obligatory gratitude to the compassion shown by the STMM team. ${ }^{20}$ They emphasised that STMMs could inadvertently contribute to inequalities in health in LMICs by adding to the complexities of a local healthcare system, which may not be accessible or affordable to all. ${ }^{20}$ Working with local healthcare providers, aiming to explore their needs and how healthcare can be improved for all should be an essential part of all STMMs. ${ }^{11}$

\section{Recommendations}

Mercy Ships and other mission-based organisations should consider how their interactions with local surgical teams and training programme are perceived by patients receiving care. For example, Mercy Ships offers clinical training for local physicians and nurses, but should expand the amount of direct interaction these clinicians get with patients. The number of participants noting poor reception of patients in local hospitals suggests additional training in the care of and interaction with patients from a non-clinical, interpersonal-focused way could be beneficial. Participants specifically appreciated that Mercy Ships staff explained everything they were doing; supporting research suggests patients who understand what is happening during a medical procedure feel much less fearful throughout the process. ${ }^{30}$ Ultimately, patient trust of local providers must be increased; through patient advocacy, so patients feel less fearful about surgery, and through provider training in trustbuilding behaviours. Missions and local surgical teams must work together to increase patient trust and improve patient experiences. A best-practices framework, such as that developed by Cheng et al, ${ }^{10}$ built on the work by Maki $e t a l,{ }^{31}$ and adapted to the organisation and environment, should be followed for each mission to ensure sustainability of care, planning of long-term impact, integration of local healthcare systems and helping local provision to become fully self-sufficient. Ultimately, the goal should be that STMMs are no longer required, and that holistic, accessible, quality care is provided locally.

\section{Limitations}

The findings of this study are presented with limitations. The sample was self-selecting and therefore not all patients' views may have been included. For example, while all participants interviewed had positive feedback and experiences with Mercy Ships, there may be patients who had poor experiences that were not reached. While participants shared they were no longer fearful of foreign surgery, there is no way of knowing how many potential patients with treatable surgical conditions never came to seek care due to fear, or were offered a surgical space but did not keep their appointment due to fear or distrust. There may also be patients in Benin who do not fear local hospitals and have received care, who were never a part of the Mercy Ships programme and therefore not included in our sampling. As discussed above, the positionality of the researcher may also have been a limitation.

\section{CONCLUSION}

Existing research on perspectives and experiences of surgical patients is limited. However, patients are a critical piece of a well-functioning surgical system, and their perspectives, beliefs and behaviours must be taken into consideration in any surgical systems' strengthening programme. This research shows Mercy Ships and other foreign mission-based surgical providers, while meeting 
an immediate need in patients' lives and leaving a positive impact therein, may be leaving behind a legacy of distrust and fear that no amount of surgical systems strengthening can overcome. Global surgery, as an emerging field of study that is expanding and growing extremely quickly, ${ }^{32}$ needs to abandon the colonial mindset and focus on systems strengthening that directly incorporates a patient's needs, satisfaction and trust in local systems; otherwise, the impact will be short-lived and unsustainable without foreign support.

\section{Twitter Floor T.E. Christie-de Jong @floorchristie}

Contributors KLC conceived the study and acquired the data. Both authors were involved in the design, data interpretation and analysis. KLC first drafted the manuscript and both authors were involved in critical revision of the article and approved the final version for publication. The data in this article were collected by $\mathrm{KLC}$ as a part of a dissertation required for achieving the Master of Public Health degree, University of Liverpool, which FTECdJ supervised.

Funding The Article Processing Charge was supported by Mercy Ships. Mercy Ships did not fund the research and had no role in the study design, data collection and analysis, decision to publish or preparation of the manuscript.

Competing interests None declared.

Patient consent for publication Not required.

Ethics approval Ethical approval for this study was granted by the Mercy Ships Institutional Review Board, the University of Liverpool and the Ministry of Health, Republic of Benin.

Provenance and peer review Not commissioned; externally peer reviewed.

Data availability statement No data are available.

Open access This is an open access article distributed in accordance with the Creative Commons Attribution Non Commercial (CC BY-NC 4.0) license, which permits others to distribute, remix, adapt, build upon this work non-commercially, and license their derivative works on different terms, provided the original work is properly cited, appropriate credit is given, any changes made indicated, and the use is non-commercial. See: http://creativecommons.org/licenses/by-nc/4.0/.

\section{REFERENCES}

1 Meara JG, Leather AJM, Hagander L, et al. Global surgery 2030: evidence and solutions for achieving health, welfare, and economic development. The Lancet 2015;386:569-624.

2 Lavy C, Sauven K, Mkandawire N, et al. State of surgery in tropical Africa: a review. World J Surg 2011;35:262-71.

3 Hoyler M, Finlayson SRG, McClain CD, et al. Shortage of doctors, shortage of data: a review of the global surgery, obstetrics, and anesthesia workforce literature. World J Surg 2014;38:269-80.

4 Kushner AL, Groen RS, Kamara TB, et al. Assessment of pediatric surgery capacity at government hospitals in Sierra Leone. World J Surg 2012;36:2554-8.

5 Henry JA, Windapo O, Kushner AL, et al. A survey of surgical capacity in rural southern Nigeria: opportunities for change. World $\mathrm{J}$ Surg 2012;36:2811-8.

6 Petroze RT, Nzayisenga A, Rusanganwa V, et al. Comprehensive national analysis of emergency and essential surgical capacity in Rwanda. Br J Surg 2012;99:436-43.

7 Ng-Kamstra JS, Greenberg SLM, Abdullah F, et al. Global surgery 2030: a roadmap for high income country actors. BMJ Glob Health 2016;1:e000011.

8 Maki J, Qualls M, White B, et al. Health impact assessment and short-term medical missions: a methods study to evaluate quality of care. BMC Health Serv Res 2008;8:121-21.
9 Shrime MG, Sleemi A, Ravilla TD. Charitable platforms in global surgery: a systematic review of their effectiveness, costeffectiveness, sustainability, and role training. World J Surg 2015;39:10-20.

10 Cheng M, Rodriguez E. Short-Term medical relief TRIPS to help vulnerable populations in Latin America. bringing clarity to the scene. Int J Environ Res Public Health 2019;16:745.

11 Sullivan N. Hosting gazes: Clinical Volunteer Tourism \& Hospital Hospitality in Tanzania. Volunteer economies: The politics and ethics of voluntary labour in Africa: James Currey Publishers, 2016: 140-63.

12 Sykes KJ. Short-Term medical service trips: a systematic review of the evidence. Am J Public Health 2014;104:e38-48.

13 Carlson LC, Hatcher KW, Vanderburg R, et al. A health systems perspective on the mission model for cleft lip and palate surgery: a matter of sustainability or responsibility? J Craniofac Surg 2015;26:1079-83.

14 Lehnerdt G, van Delden A, Lautermann J. Management of an "Ear Camp" for children in Namibia. Int J Pediatr Otorhinolaryngol 2005;69:663-8.

15 Cheng H, Webster F, Platt J, et al. Patient-Reported outcomes (pros) in surgical humanitarian missions to low- and middle-income countries: a scoping review. Clinics in Surgery 2016;1.

16 Sharp HM, Canady JW, Ligot FAC, et al. Caregiver and patient reported outcomes after repair of cleft lip and/or palate in the Philippines. Cleft Palate Craniofac J 2008;45:163-71.

17 Epstein RM, Street RL. The values and value of patient-centered care: Annals family Med. , 2011: 9, 100-3.

18 Stewart M, Brown JB, Donner A, et al. The impact of patientcentered care on outcomes. J Fam Pract 2000;49:796-804.

19 White M, Alcorn D, Randall K, et al. Evaluation of patient satisfaction, impact and Disability-Free survival after a surgical mission in Madagascar: a pilot survey. World J Surg 2017;41:364-9.

20 Roche S, Brockington M, Fathima S, et al. Freedom of choice, expressions of gratitude: patient experiences of short-term surgical missions in Guatemala. Soc Sci Med 2018;208:117-25.

21 Ships M, ed. Benin 2016-2017 Final Narrative Report, 2017.

22 Lin BM, White M, Glover A, et al. Barriers to surgical care and health outcomes: a prospective study on the relation between wealth, sex, and postoperative complications in the Republic of Congo. World $J$ Surg 2017;41:14-23.

23 Kessy JP, Lewallen S. Poverty as a barrier to accessing cataract surgery: a study from Tanzania. Br J Ophthalmol 2007;91:1114-6.

24 Grimes CE, Bowman KG, Dodgion CM, et al. Systematic review of barriers to surgical care in low-income and middle-income countries. World J Surg 2011;35:941-50.

25 van Loenhout JAF, Delbiso TD, Gupta S, et al. Barriers to surgical care in Nepal. BMC Health Serv Res 2017;17:72.

26 White MC, Hamer M, Biddell J, et al. Facilitating access to surgical care through a decentralised case-finding strategy: experience in Madagascar. BMJ Global Health 2017;2. e000427.

27 GE. GE Foundation Commits \$25 million to Global Safe Surgery Initiative, 2015. Available: http://www.genewsroom.com/pressreleases/ge-foundation-commits-25-million-global-safe-surgeryinitiative-281817 [Accessed 12 Sep 2017].

28 Lee $Y-Y, L$ Lin J. Linking patients' trust in physicians to health outcomes. Br J Hosp Med 2008;69:42-6.

29 Bouchard M, Kohler JC, Orbinski J, et al. Corruption in the health care sector: a barrier to access of orthopaedic care and medical devices in Uganda. BMC Int Health Hum Rights 2012;12:5.

30 Nijkamp MD, Ruiter RAC, Roeling M, et al. Factors related to fear in patients undergoing cataract surgery: a qualitative study focusing on factors associated with fear and reassurance among patients who need to undergo cataract surgery. Patient Educ Couns 2002;47:265-72.

31 Maki J, Qualls M, White B, et al. Health impact assessment and short-term medical missions: a methods study to evaluate quality of care. BMC Health Serv Res 2008;8:121.

32 Debas HT. The emergence and future of global surgery in the United States. JAMA Surg 2015;150:833-4. 